\title{
Incidence of different morphological types of diabetic macular edema on OCT in western Indian Population
}

\author{
Nilesh Sharmali ${ }^{1}$, Minhaz Karkhanawala ${ }^{2}$, Somesh Aggarwal $^{3 *}$, Anindita Duttaroy ${ }^{4}$, Jignasha Makvana $^{5}$ \\ ${ }^{\mathbf{1 , 4}, \mathbf{5}}$ Resident, ${ }^{\mathbf{2}}$ Senior Resident, ${ }^{3}$ Professor and Head of Retina Unit, M and J Western Regional Institute of Ophthalmology Ahmedabad, \\ Gujarat, India
}

\begin{abstract}
Objective: This study is aimed at establishing the incidence of different morphological types of diabetic macular edema on OCT in the Western Indian population.

Design: The study was a retrospective, observational, cross-sectional study based on hospital records.

Setting: $M$ and J Western Regional Institute of Ophthalmology, Civil Hospital, Ahmedabad, India.

Subjects: 206 eyes of 105 patients were studied, out of which 65 were men (61.90\%) and 40(39.10\%) were women. The age of patients ranged from 30-80 years (mean 56 years).

Intervention: No intervention was done for the purpose of the study.

Main outcome and Measures: The incidence of different morphological types of macular edema on OCT was calculated.

Results: The incidence of various types of ME was same across all age groups as well as both gender groups. The incidence was as follows: no macular edema in $18.93 \%$ patients, focal macular thickening in $13.1 \%$, diffuse non cystoid macular edema in $12.13 \%$, diffuse cystoid macular edema in $24.75 \%$, tractional macular edema due to posterior hyaloid traction in $2.91 \%$, tractional edema due to epiretinal membrane in $8.73 \%$, both posterior hyaloid traction and epiretinal membrane in $3.39 \%$ and macular serous retinal detachment in $16 \%$ patients.

Conclusion: With the availability of the geographical distribution of different types of DME, treatment of DME can be tailored to the population. Also, the causes of such a difference in incidence of various patterns of DME in different populations can be established with further studies.
\end{abstract}

Keywords: Diabetic macular edema (DME), Optical coherence tomography (OCT).

\section{Introduction}

Diabetic macular edema is one of the major causes of visual impairment in individuals with diabetes. ETDRS defined diabetic macular edema (DME) as focal or diffuse retinal thickening in the macular area. When this thickening involves or threatens the fovea, it is defined as clinically significant and treatment is indicated to reduce progressive visual loss. The risk factors for DME are largely similar to DR, but dyslipidemia appears to play a more significant role. Modifiable risk factors for DME include hyperglycemia, hypertension, dyslipidemia and inflammation. Non modifiable risk factors include ethnicity, duration of diabetes and pregnancy. ${ }^{1}$ Variation in the prevalence of these factors among sub populations may be responsible for difference in the morphology of DME leading to visual loss in patients with diabetes mellitus. Traditional methods of assessing DME include contact and non contact slit lamp biomicroscopy, indirect fundoscopy, flourescein angiography and fundus stereo-photography. Ten years after ETDRS, Optical Coherence Tomography (OCT) greatly enhanced our ability to detect macular thickening objectively and has brought new insights on the morphology of edema and on the presence of vitreoretinal interface abnormalities. It demonstrates that macular edema is a complex clinical entity with various morphologies that have to be precisely described in order to choose the correct therapeutic approach and understand its potential benefits. OCT has also become the preferred indicator of therapeutic benefit through a precise measurement of variation in retinal thickness and condition of the vitreomacular interface. The geographical distribution of morphological patterns of DME has never been studied previously. This study attempts to find out the incidence of various types of DME in the western Indian population. With the availability of such data, treatment of DME can be tailored according to the distribution of various types of DME in a given geographical area. Such a study also serves as a basis for study of the role of various genetic and environmental factors instrumental in the development of various patters of DME in a given population.

\section{Subjects and Methods}

In a retrospective, observational study, 206 eyes of 105 diabetic patients at a tertiary eye institute in Western India were studied. The review of medical records of all patients included the age of the patient, fundus photograph and spectral domain OCT reports. Inclusion criteria were type 1 or type 2 diabetes mellitus and 'clinically significant' DME on fundus photograph. Eyes with significant media opacities resulting in poor OCT signal strength were

\footnotetext{
*Corresponding Author: Somesh Aggarwal, M and J Western Regional Institute of Ophthalmology Ahmedabad, Gujarat, India Email:dr.somesh@yahoo.com
} http://doi.org/10.18231/j.ijooo.2019.036 
excluded. Following parameters were evaluated on SDOCT: retinal thickness- central foveal thickness, retinal thickness in ETDRS zones, average retinal thickness, volume, and morphology and epiretinal traction. Different morphological patterns of macular edema were described and their incidence calculated.

Statistical analysis was performed to calculate the prevalence of different patterns of DME on OCT. The variables studied were the incidence of different types of macular edema on SD-OCT.

\section{Results}

65 men $(61.90 \%)$ and 40(39.10\%) women were included in this study. The age of patients ranged from 30-80 years (mean 56 years). The incidence of different morphological types of macular edema was as following:

Table 1: Incidence of different morphological types of diabetic macular edema on OCT

\begin{tabular}{|l|c|c|}
\hline \multicolumn{1}{|c|}{ Morphological type of DME } & $\begin{array}{c}\text { Number of } \\
\text { patients }\end{array}$ & $\begin{array}{c}\text { Percentage } \\
(\%)\end{array}$ \\
\hline 1. None & 39 & 18.93 \\
\hline 2. Focal Macular Thickening & 27 & 13.1 \\
\hline $\begin{array}{l}\text { 3. Diffuse Non-Cystoid } \\
\text { Macular Edema }\end{array}$ & 25 & 12.13 \\
\hline $\begin{array}{l}\text { 4. Diffuse Cystoid Macular } \\
\text { Edema }\end{array}$ & 51 & 24.75 \\
\hline 5. Tractional Macular Edema & & \\
\hline $\begin{array}{l}\text { 5a. Posterior Hyaloid } \\
\text { Traction }\end{array}$ & 6 & 2.91 \\
\hline $\begin{array}{l}\text { 5b. Epiretinal Membrane } \\
\text { 5c. Both Posterior Hyaloid } \\
\text { And Epiretinal Membrane }\end{array}$ & 7 & 8.73 \\
\hline $\begin{array}{l}\text { 6. Macular Serous Retinal } \\
\text { Detachment }\end{array}$ & 33 & 16 \\
\hline
\end{tabular}

The incidence of various types of ME was same across all age groups as well as both gender groups.

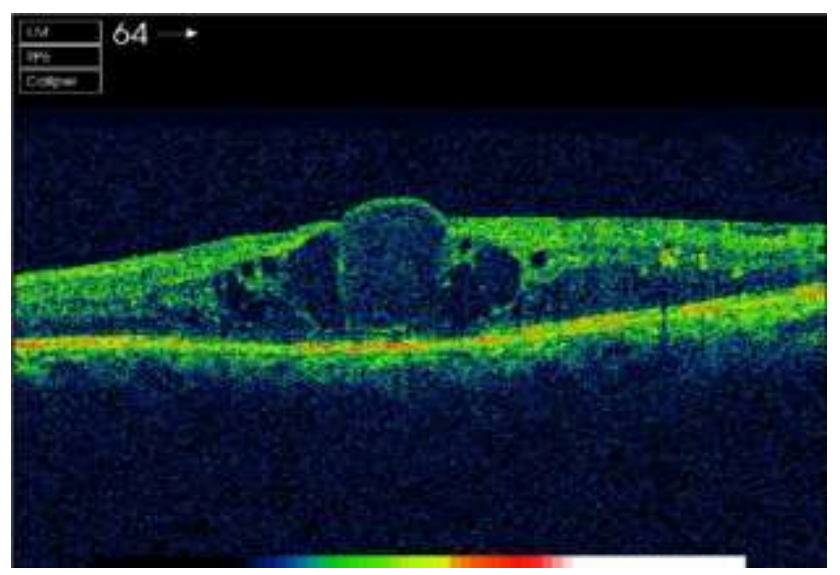

Fig. 1: Cystoid macular edema

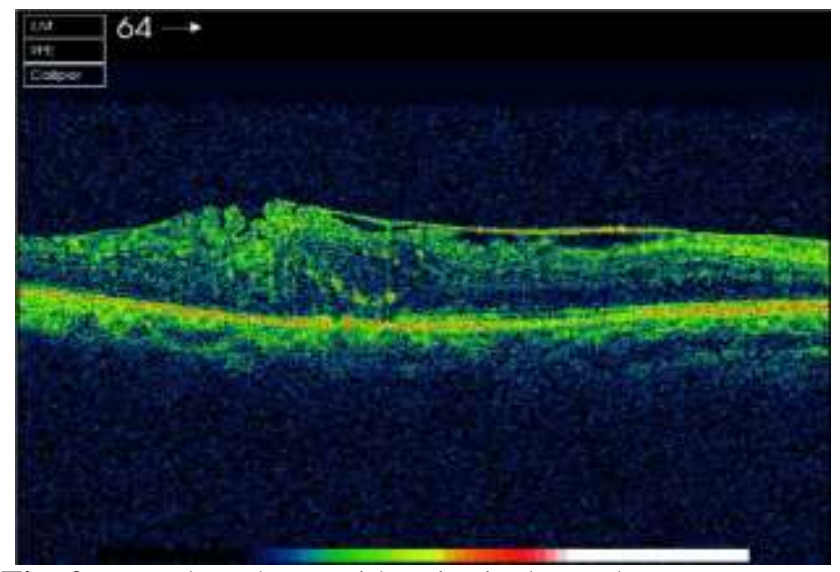

Fig. 2: Macular edema with epiretinal membrane

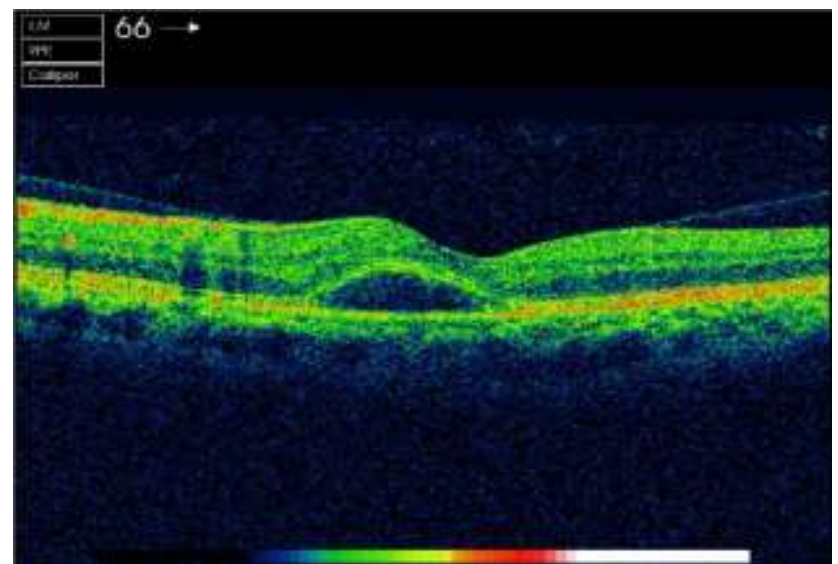

Fig. 3: Macular serous detachment

\section{Discussion}

Macular edema remains the most common reason for intervention by ophthalmologists in patients with diabetes confronting visual loss.,3 Measuring macular edema is therefore of major importance in imaging patients with DME. ${ }^{4}$ OCT analysis demonstrates that DME is a complex entity with different patterns, extent, progression, and possible abnormal vitreoretinal relationships. Considering the emergence of new therapeutic modalities, such as intravitreal corticosteroids and anti-VEGF injections or vitrectomy for DME, it seems worthwhile to propose a detailed and precise tomographic classification of DME. ${ }^{3,5}$

Three OCT patterns of DME were reported by Otani and associates in $1999^{6}$ : sponge like swelling (88\%), retinal edema with cystic spaces $(47 \%)$ and retina edema with subfoveal fluid accumulation (15\%).

In 2004, Kang proposed a classification in 4 types $^{7}$ : type 1 thickening with homogenous optical reflectivity $(55.2 \%)$, type 2 thickening with markedly decreased optical reflectivity in the outer retinal layers $(30.3 \%)$, type $3 \mathrm{~A}$ foveolar detachment without traction (14.5\%) and type 3B foveolar detachment with apparent vitreofoveal traction (2.8\%). In this classification, authors considered the tractional factor and subdivided the foveal detachment according to the presence of tractional membrane. These 
observations have important therapeutic impacts.

Another OCT based classification was published in 2006 by Kim and associates ${ }^{8}$ : diffuse retinal thickening $(97 \%)$, cystoids macular edema $(55 \%)$, and serous retinal detachment $(2.9 \%)$.

In all these classifications, foveal macular edema was omitted. Hence a new OCT classification was proposed in 2012 by Afif Maalej and associates that classified DME in 5 types ${ }^{9}$ : focal macular thickening (30.3\%), diffuse macular edema without cysts $(20.9 \%)$, cystoid macular edema $(35.7 \%)$, tractional macular edema $(7.1 \%$ due to posterior hyaloids traction, $6 \%$ due to epiretinal membrane and $3.2 \%$ due to a combination of both) and serous retinal detachments $(8.6 \%)$. Each of the morphological types of DME represents a distinct entity that requires specific treatment regimens to achieve the best final result. Tractional macular detachments are the best candidates for vitrectomy.

Following is the comparison between the incidences of DME in our study as compared to the study by Afif Maalej et al.

Table 2: Comparison of incidence of DME in our study compared to study by Afif Maalej et al.

\begin{tabular}{|l|c|c|}
\hline $\begin{array}{c}\text { Morphological type } \\
\text { of DME }\end{array}$ & $\begin{array}{c}\text { Percentage } \\
(\mathbf{\%})\end{array}$ & $\begin{array}{c}\text { Percentage of } \\
\text { patients in study by } \\
\text { Afif Maalej et al (\%) }\end{array}$ \\
\hline 1. None & 18.93 & 5.5 \\
\hline $\begin{array}{l}\text { 2. Focal macular } \\
\text { thickening }\end{array}$ & 13.1 & 30.3 \\
\hline
\end{tabular}

Based on the comparison above, it might be deduced that the incidence of focal macular thickening, diffuse non cystoids macular edema, diffuse cystoids macular edema and tractional macular edema due to posterior hyaloids traction was less as compared to the study by Maalej et al. however, the incidence of epiretinal membrane as well as macular serous retinal detachment was significantly higher than the other study. The difference in the incidence as mentioned above was clinically significant. Keeping in mind that the study by Maalej et al was conducted at a centre in Tunisia, this difference in morphology might be an outcome of a variety of factors.

The prevalence of DME in patients with diabetes in various populations has been studied in Various studies. The results of these studies show that there is often a significant variation in the prevalence of DME in different subpopulations. Such differences may be due to several risk factors differing in different subpopulations. The same may apply to the patterns of macular edema in different subpopulations. The patterns of macular edema might vary with different populations based on the distribution of risk factors in the respective population. Such risk factors include modifiable as well as non modifiable risk factors. However, the patterns of macular edema in the Indian population have never been previously studied. As seen in the observations above, 67.91 percentage of patients in our study had DME involving the fovea without the presence of vitreoretinal traction. 15.03 percentage of patients had DME with vitreoretinal traction. The former patients are best candidates for medical treatment of DME whereas the latter are candidates for vitrectomy. A detailed study of various factors influencing the incidence of different types of macular edema is required for further explanation of the above difference.

The vast difference in their incidence might be used as a clinical tool in guiding treatment for DME. With majority of patients not having a tractional component responsible for the macular edema, medical management in the form of intravitreal anti-VEGF as well as laser are indicated in absence of the availability of OCT. However, any suspicion of tractional DME should be confirmed by OCT and the treatment tailored accordingly.

\section{Conclusion}

With the widespread availability of OCT, it is now an essential tool to evaluate and classify DME morphologically. The patterns of DME may vary across different subpopulations; further studies are required to substantiate the evidence as well as evaluate the risk factors for such variation. A tailored approach of treatment would ensure the best final result in patients in terms of visual acuity as well as morphological evidence of response to treatment. With the availability of the geographical distribution of different types of DME, treatment of DME can be tailored.

\section{Source of Funding}

None.

\section{Conflict of Interest}

None.

\section{References}

1. Ding, J\& Wong, T-Y. Current epidemiology of diabetic retinopathy and diabetic macular edema. Currt Diabetes Rep 2012;12:346-354.

2. Buabbud JC, Al-latayfeh MM, Sun JK. Optical coherence tomography imaging for diabetic retinopathy and macular edema. Curr Diab Rep 2010;10:264-269

3. Massin P, Girach A, Erginay A, Gaudric A. Optical coherence tomography: a key to the future management of patients with diabetic macular oedema. Acta Ophthalmol Scand 2006;84:466-74

4. Browning J. Interpreting thickness changes in the diabetic macula: the problem of short term variation in optical coherence tomography- measured macular thickening. Trans Am Ophthalmol Soc 2010;108:62-76

5. Funatsu H, Yamashita H, Shimizu E, Mimurac T, Nakamura $\mathrm{S}$, Quantitative measurement of retinal thickness in patients with diabetic macular edema is useful for evaluation of therapeutic agents. Diabetes Res Clin Pract 2004;66:219-27.

6. Otani T, Kishi S, Maryyama Y. Patterns of diabetic macular edema with optical coherence tomography. Am J Ophthalmol 127:688-693

7. Kang SW, Park CY, Ham DI. The correlation between flourescein angiographic and optical coherence tomographic features in clinically significant diabetic macular edema. Am J 
Ophthalmol 2004;137:313-22.

8. Kim BY, Smith SD, Kaiser PK. Optical Coherence tomographic patterns of diabetic macular edema. Am J Ophthalmol 2006;142:405-12

9. Maalej A, Cheima W, Asma K, Salem G. optical coherence tomography for diabetic macular edema: Early Diagnosis, classification and quantitative assessment. J Clin Exp Ophtalmol 2012;S2:004.

How to cite this article: Sharmali N, Karkhanawala M, Aggarwal S, Duttaroy A, Makvana J. Incidence of different morphological types of diabetic macular edema on OCT in western Indian Population, Int J Ocul Oncol Oculoplasty 2019;5(3):139-142. 